\title{
Decomposition of biospeckle images in temporary spectral bands
}

\author{
G. H. Sendra \\ Laboratorio Láser, Facultad de Ingeniería, Universidad Nacional de Mar del Plata, Juan B. Justo 4302 ,
} Mar del Plata, Argentina

\section{R. Arizaga, H. Rabal, and M. Trivi}

Centro de Investigaciones Opticas, Consejo Nacional de Investigaciones Científicas y Técnicas and UID Optimo, Comisión de Investigaciones Científicas de Provincia de Buenos Aires, Facultad de Ingenieria, Universidad Nacional de La Plata, Casilla Correo 124, 1900 La Plata, Argentina

Received December 14, 2004

We present a method of analysis of images of dynamic speckle based on the filtering in frequency bands of the temporary history of each pixel. Butterworth filters are applied to the temporary evolution, and different images are constructed showing the energy in each frequency band. Different degrees of activity of the sample in study, presumably attributed to different origins, are found. The method is exemplified with images of bruising damage in fruits and of biological activity in germinating corn seeds. It is found that the activity in the bruised region of an apple differs from the activity of healthy regions in a certain characteristic frequency range. The activity of the embryo can also be distinguished from that of the endosperm in corn seeds during germination. (C) 2005 Optical Society of America

OCIS codes: $100.0100,120.6150,000.1430$.

Dynamic speckle or biospeckle is observed in biological samples illuminated by laser light. The properties and applications of this phenomenon have been treated in the literature. ${ }^{1-3}$

There are several methods for display of speckle pattern activity. Laser speckle contrast analysis, ${ }^{4}$ proposed by Briers and Webster, uses the local contrast analysis of the integrated time-varying speckle in a nonscanning full-field technique for monitoring capillary blood flow. In the method of Fujii et al., ${ }^{5}$ an image display based on the accumulation of differences between images divided by its average shows a microcirculation map of human skin surface. Konishi and $\mathrm{Fujii}^{6}$ used the signal-to-noise ratio in a realtime application to visualize a blood flow map of the human retina. The method of Arizaga et $\mathrm{al}^{7}{ }^{7}$ is based on accumulating all possible differences between consecutive and nonconsecutive frames for each pixel of images in a seed analysis application. Recently other approaches were presented to characterization of atherosclerotic plaque by spatial and temporal speckle pattern analysis ${ }^{8}$ and to applying timedependent speckle in holographic optical coherence imaging to the study of tumor tissue. ${ }^{9}$

In the aforementioned methods, the result is a single image that shows a certain measure of the total activity of the sample. In this Letter we propose a method with which to analyze biospeckle activity based on decomposition of images in temporary spectral bands that permits us to obtain several measures with a better-defined meaning, thus adding to a better-detailed characterization of the behavior of the samples.

Most algorithms already developed for activity images discard time information and pool all the information concerning activity measurement in a single measure. Biological phenomena do not exhibit well- defined frequencies, but, in some cases, they differ in the frequency ranges where energy is concentrated. By using the filters in the frequency domain, one can discriminate phenomena in the sample that occur on different time scales. We present some examples to illustrate the methodology, but this technique can be extended to other applications of dynamic speckle in biological samples.

We consider biospeckle image sequences of objects in regions in which different activities are present. It can be observed that the levels of intensity variation of the speckle grains are different in each region. It is possible to find pixels that show fast intensity variations and others that show slow ones or no variations at all. To study these variations and characterize typical times of different regions we can state the analysis in frequencies instead of times.

Fujii et al. ${ }^{10}$ applied this idea to the measurement of blood flow at a point. We extend its use to a sequence of images. Instead of carrying out a Fourier analysis, we use a bank of filters, implemented in a computer, to find bands that depict different types of activity. In this way, a region with a certain type of activity can be associated with one or several frequency bands.

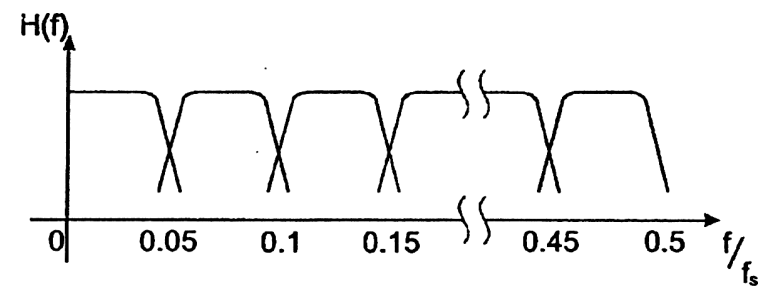

Fig. 1. Frequency bands in the Butterworth filters bank. 
We recorded a sequence of $N$ successive speckle images with a sampling frequency $f_{s}$. In this way it was possible to observe how a pixel evolves through the $N$ images. That evolution can be treated as a time series and can be processed in the following way: Each signal corresponding to the evolution of every pixel was used as input to a bank of filters. The intensity values were previously divided by their temporal mean value to minimize local differences in reflectivity or illumination of the object. The maximum frequency that can be adequately analyzed is determined by the sampling theorem and is half of sampling frequency $f_{s}$. The latter is set by the CCD camera, the size of the image, and the frame grabber. The bank of filters is outlined in Fig. 1. In our case, ten $5^{\circ}$ order Butterworth ${ }^{11}$ filters were used, but this number can be varied according to the required discrimination. The bank was implemented in a computer using Matlab software. We chose the Butterworth filter because, in addition to its simplicity, it is maximally flat. Other filters, an infinite impulse response, or a finite impulse response could be used.

By means of this bank of filters, ten corresponding signals of each filter of each temporary pixel evolution were obtained as output. Average energy $E_{b}$ in each signal was then calculated:

$$
E_{b}=\sum_{n=1}^{N} p_{b}^{2}(n),
$$

where $p_{b}(n)$ is the intensity of the filtered pixel in the $n$th image for filter b divided by its mean value and $N$ is the total number of images. In this way, ten values of energy for each pixel were obtained, each of them belonging to one of the frequency bands in Fig. 1.

With these values it is possible to build ten images of the active object, each one of which shows how much energy of time-varying speckle there is in a certain frequency band. False color assignment to the gray levels in the results would help in discrimination.

Using the proposed method, we performed two experiments: studies of bruising damage in fruits and of biological activity in corn seeds. In the experiments the sample was illuminated by an expanded low-power (10 $\mathrm{mW}) \mathrm{He}-\mathrm{Ne}$ laser. By using a CCD camera as a detector and a host computer with a frame grabber, we registered, digitized, and stored the successive sample images. Care was taken that the speckles were well resolved by the CCD sensor.

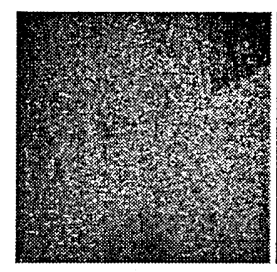

(a)

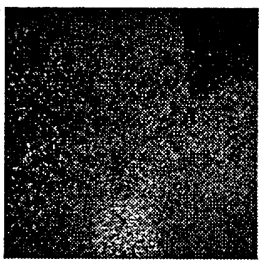

(b)

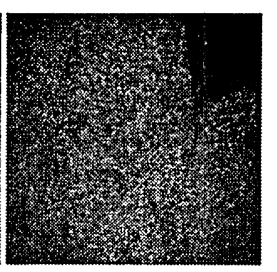

(c)
Fig. 2. Results of the apple bruising experiment: (a) first band $(0-0.125 \mathrm{~Hz})$, (b) third band $(0.250-0.375 \mathrm{~Hz}),(\mathrm{c})$ tenth band $(1.125-1.250 \mathrm{~Hz})$

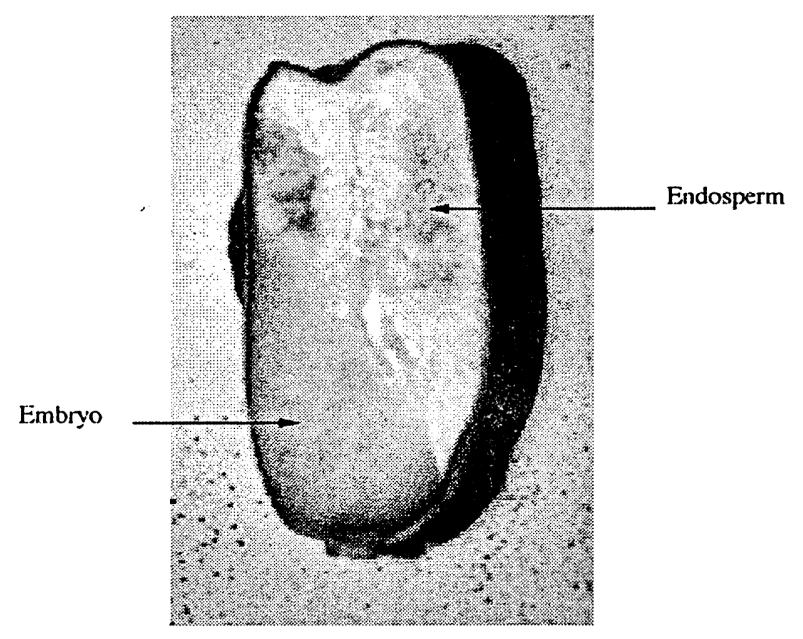

Fig. 3. Incoherent image of a corn seed.

We used a low illuminating intensity to minimize the effect of irradiation on the sample activity. The mean laser illumination was kept constant during all measurements, and no appreciable changes in sample reflectivity were observed.

An apple was bruised ${ }^{12}$ by dropping a sphere (diameter, $21.9 \mathrm{~mm}$; weight, $133.6 \mathrm{~g}$ ) on it from a controlled height $(h=20 \mathrm{~cm})$. The bruised region could not be distinguished from the unbruised rest of the apple by visual inspection.

A series of images of dynamic speckle was registered after the blow and the images were processed with the proposed algorithm. An inert object was included as a reference in the upper-right corner of each image. In Fig. 2 we show three representative results. Figure 2(a) corresponds to the lowerfrequency band $(0-0.125 \mathrm{~Hz})$. The dark zone below represents the bruised region. This means that the activity in this zone and for this frequency band is very low, slightly higher than in an inert region but lower than in a healthy region. Conversely, Fig. 2(b), corresponding to the third band $(0.250-0.375 \mathrm{~Hz})$, shows the bruised region with higher activity than in the rest of the apple. Band 10 [1.125-1.250 Hz; Fig. 2(c)] does not show any difference between bruised and healthy regions.

The technique was then applied to images of speckle in corn seeds that had previously been hydrated to start the germination process and had been longitudinally cut in halves. In Fig. 3 an image of seed illuminated by incoherent light is exhibited; the embryo and the endosperm regions can be observed. The six most significant resultant images that correspond to the energy in each band are shown in Fig. 4.

The image of the first band $(0-0.1 \mathrm{~Hz})$ shows the bright zone of the endosperm. The embryo, which is the active zone in germination, is dark here because this band emphasizes only the zones of slower activity. The last image represents the highest frequency band reported here $(0.5-0.6 \mathrm{~Hz})$. The embryo is bright here, and the endosperm is dark. That is, the embryo shows higher activity in the high frequencies. For higher frequencies than that the results do not show significant differences. 


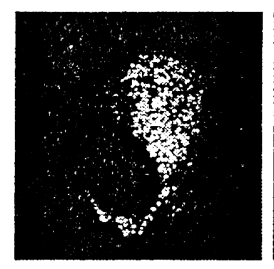

(a)

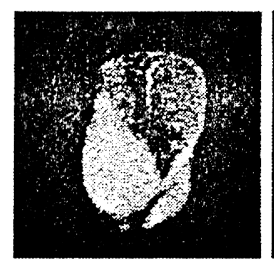

(d)

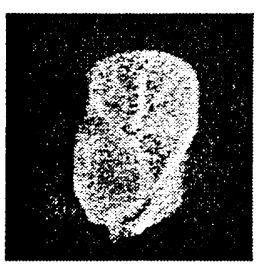

(b)

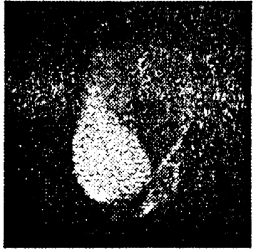

(e)

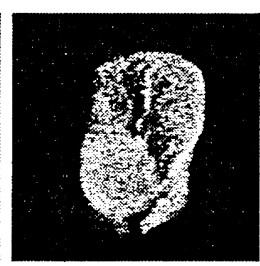

(c)

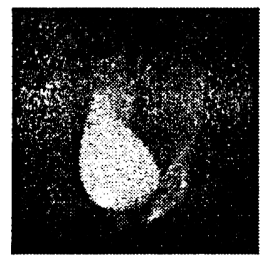

(f)
Fig. 4. Results of the corn seed experiment: (a) first band $(0-0.1 \mathrm{~Hz})$, (b) second band $(0.1-0.2 \mathrm{~Hz})$, (c) third band $(0.2-0.3 \mathrm{~Hz})$, (d) fourth band $(0.3-0.4 \mathrm{~Hz}),(\mathrm{e})$ fifth band $(0.4-0.5 \mathrm{~Hz}),(\mathrm{f})$ sixth band $(0.5-0.6 \mathrm{~Hz})$.

In the intermediate-frequency bands [Figs. $4(\mathrm{~b})-4(\mathrm{e})$ ], other regions, such as a riverlike trace in the endosperm, appear bright, probably due to a break but the biological origin of the brightness is not yet certain. It is apparent that in the intermediatefrequency bands the method permits the estimation of the energy that corresponds to several time scales. Several regions can be discriminated by differences in activity.

Adequate interpretation of the meaning of these measures requires learning through experiment to evaluate which activities are normal and which interfere with germination. In these images there seems to be considerable biological information, but its discussion is beyond the scope of this work.

In conclusion, we have proposed the use of temporal filters to identify regions with speckle activity of different possible causes. A bank of filters has been used, and different types of activity have been detected. In this way, regions of the object that show certain types of activity can be associated with one or several frequency bands.

Applications to corn seeds of the onset of germination and to a bruised apple were used as examples. In both cases it was possible to discriminate regions with different types of activity. The interval of frequencies where the apple's bruised region can be dis- tinguished from the healthy regions was identified, and the activities of the embryo and the endosperm in corn seeds were explored.

Compared with existing methods for activity assessment, this method is both quantitative and qualitative as it provides visual information on the several time scales where the various phenomena occur without loss of spatial resolution. Additional information is then obtained, as in all the other displays all the activity information is pooled in a single image. Unfortunately, in its present state the method cannot be implemented in real time.

The technique can be used to segment other types of phenomena in biological samples or in industrial processes (corrosion, drying of paint, vibrations, etc). Each application requires learning and expertise in the phenomenon involved and provides information on the times scales that determine the activity of each region.

This study was partially supported by grants from the Consejo Nacional de Investigaciones Científicas y Técnicas, the Comisión de Investigaciones Científicas de Provincia de Buenos Aires, the Facultad de Ingeniería, Universidad Nacional de La Plata; and the Facultad de Ingeniería, Universidad Nacional de Mar del Plata, Argentina. H. Rabal's e-mail address is hrabal@ing.unlp.edu.ar.

\section{References}

1. Y. Aizu and T. Asakura, in Trends in Optics, A. Consortini, ed. (Academic, 1996), Chap. 2.

2. T. Okamoto and T. Asakura, in Progress in Optics, E. Wolf, ed. (Elsevier Science, 1995), Vol. XXXIV, pp. 185.

3. J. D. Briers, Opt. Eng. 32, 277 (1993).

4. J. D. Briers and S. Webster, J. Biomed. Opt. 1, 174 (1996).

5. H. Fujii, K. Nohira, Y. Yamamoto, H. Ikawa, and T. Ohura, Appl. Opt. 26, 5321 (1987).

6. N. Konishi and H. Fujii. Opt. Eng. 34, 753 (1995).

7. R. Arizaga, N. Cap, H. J. Rabal, and M. Trivi, Opt. Eng. 41, 287 (2002).

8. G. J. Tearney and B. E. Bouma, Opt. Lett. 27, 533 (2002).

9. P. Yu, I. Peng, M. Mustata, J. J. Turek, M. R. Melloch, and D. D. Nolte, Opt. Lett. 29, 68 (2004).

10. H. Fujii, T. Asakura, K. Nohira, Y. Shintomi, and T. Ohura, Opt. Lett. 10, 104 (1985).

11. A. V. Oppenheim and R. W. Schafer, Digital Signal Processing (Prentice-Hall, 1975), pp. 211.

12. M. Pajuelo, G. Baldwin, H. J. Rabal, N. Cap, R. Arizaga, and M. Trivi, Opt. Lasers Eng. 40, 13 (2003). 